\title{
AcneNet - A Deep CNN Based Classification Approach for Acne Classes
}

\author{
Masum Shah Junayed ${ }^{1}$, Afsana Ahsan Jeny ${ }^{1}$, Syeda Tanjila Atik ${ }^{1}$, Nafis Neehal ${ }^{1}$ \\ Asif Karim ${ }^{2 *}$, Sami Azam², Bharanidharan Shanmugam ${ }^{2}$ \\ ${ }^{1}$ Daffodil International University, Dhaka, Bangladesh \\ ${ }^{2}$ Charles Darwin University, NT, Australia \\ E-mail: ${ }^{1}$ junayed15-5008, ahsan15-5278, syeda.cse, nafis.cse\}@diu.edu.bd \\ ${ }^{2}\{$ asif.karim, sami.azam, bharanidharan.shanmugam\}@cdu.edu.au
}

Pre-print

\begin{abstract}
Skin diseases are very common and nowadays easy to get remedy from. But, sometimes properly diagnosing these diseases can be quite troublesome due to the stiff hard-todiscriminate nature of the symptoms they exhibit. Deep Neural Networks, since its recent advent, has started outperforming different algorithms in almost every sectors. One of the problem domains, where Deep Neural Networks are really thriving today, is Image Classification and Object and Pattern Discovery from images. A special type of Deep Neural Network is Convolutional Neural Networks (CNN), which are being extensively used for different sorts of computer vision and image classification related problems. Hence, we have proposed a novel approach, where we have developed and used a Deep Residual Neural Network model for classifying five classes of Acnes from images. Our model has achieved an approximate accuracy as much as $99.44 \%$ for one class, and the rest were also above $94 \%$ with fairly high precision and recall score.
\end{abstract}

Keywords-Acne diseases, Artificial Intelligence, CNN, Deep Residual Neural Network

\section{INTRODUCTION}

Acne is a common skin disorder or a skin condition which usually occurs because of the clogging of sebaceous glands in the skin along with the hair shafts in addition to the bacterial infection [1]. It can be characterized by the skin with scaly red skin (seborrhea), blackheads and whiteheads (comedones), pinheads (papules), large papules (nodules), pimples and scarring [2]. In the severe stage, large pimples start to produce firm swellings deep under the skin and become inflamed resulting in developing into painful lumps which leads to scarring [1]. At least $85 \%$ of people in the U.S. experience acne between the ages of 12 and 24 years [3]. Sufferers often experience emotional and social disturbance because of the discomfort and embarrassment that is brought by acne. Acne arises commonly during adolescence, affecting about $80-90 \%$ of teenagers in the Western world and lower rates are reported in rural societies [4]. Females are more likely to have acne than males, around $9.8 \%$ of females compared to around $9 \%$ of males [5]. Multiple types of acne lesions can occur at the same time in a person's face. Early and precise detection of those specific conditions is essential for prescribing proper treatment and improving healthcare [6]. Therefore identification of acne types has become an important and widely researched problem in the area of dermatology [7].
The traditional diagnosis method referred to as manual observation and acne calculation is labor-intensive, timeconsuming and subjective to the expert's experience and ability [8]. Also, a long training period is required in this method. To overcome this problem a variety of image processing and machine learning techniques are recently used to accurately identify the skin problems arising from acne in the human face [9]. VISIA is a widely used commercial instrument which aims to identify the acne types in the skin during cosmetic surgery. It is done by examining multispectral images [10]. However, it is very expensive and there is a need for outlining the region of interest (ROI) manually by the dermatologist during the inspection which is also very unreliable. This is the same case for most skin analysis systems nowadays.

In this paper, we have proposed a new Convolutional Neural Network (CNN) [16] model "AcneNet" which is based on Deep Residual Neural Network [17]. In the last few years, Deep Residual Neural Network [17] was the most serious work of computer vision or deep learning community.

Our proposed model "AcneNet" is a collection of many paths of differing lengths. Residual network training seems to activate very deep network by leveraging only small paths. But those paths do not depend on each other. Finally, and most interesting aspect, is that the maximum-paths are less than 1 , and only small paths are required during the training. On the other hand, longer path cannot contribute at the time of training. Thus, the remaining networks do not solve invisible gradient problems by saving gradients across the entire depth of the network. As our model related to the Residual Neural network so for its identity block it can skip a similar layer. This is why it is able to complete the work at a decent pace, simultaneously provide an effective result quickly. Figure 3 has been designed as a block diagram so that the parameters required in each layers can easily be visualized to the user. The detail steps have also been discussed in Section III.

We have used 5 classes of Acne diseases for our experiment - Closed Comedo, Cystic, Keloidalis, Open Comedo and Pustular. The remainder of the paper is sorted out as pursues: In Section II, we have given the comparison with some related papers. Section III, we have given the work procedure, described the dataset, data preprocessing, our proposed model with its training and also the Confusion Matrix for multiple class in step by step. In Section IV, we have provided the performance analysis regarding our proposed model with other CNN [16] models. 


\section{LITERATURE REVIEW}

Some previous works related to our work are described as follows-

Fuji et al. was able to distinguish several types of skin lesions such as comedo, reddish papule, pustule, and scar by using a multispectral image (MSI). However, the process still requires manual inspection which is highly unreliable [11].

Alamdari et al. [12] proposed two-level k-means clustering with the HSV model for skin lesion segmentation after processing the close-up pictures taken by a mobile phone which achieved an average accuracy of $70 \%$. Here fuzzy-c-means and Support Vector Machine (SVM) were used to separate acne scarring from active inflammatory lesions having $80 \%$ and $66.6 \%$ accuracy respectively.

Phillips et al. [13] used the principle of polarized light photography to observe and count the comedo and acne. Polarized light coming from electric and magnetic fields further vibrated in a single plane. Skin features along with color, lighting, and framing are boosted in the polarized photo in order to find the acne certainly.

In our paper, we presented a new model of CNN [16] which is based on Deep Residual Neural Network [17]. The original idea of the residual neural network exhibits a socalled "identity shortcut connection" that exceeds one or more levels. We are the first in attempting to use the feature as it has not been done yet. Majority of the works on Acne diseases have been completed without $\mathrm{CNN}$ [16]. On the contrary, CNN based models have constantly produced higher accuracy than other works. As our era is gradually introducing artificial intelligence to newer technologies such as Blockchain [22], [24] as well as many other traditional sectors [23], so we believe that our work can play a significant role.

\section{RESEARCH METHODOLOGY}

In this section, the remaining part is as follows: first, we provide the system architecture which is the main procedure of our experiment; second, we give a short description of the tools and also the CNN [16] layers which we have used; third, the datasets have been described; fourth, we discuss the pre-processing of our dataset; fifth, the proposed model with architecture has been illustrated; sixth, we discuss the trained model with train, test and validation split; finally, the binary and multiple class confusion matrices have been discussed. The following system architecture (Fig. 1) represents the main procedure of our experiment.

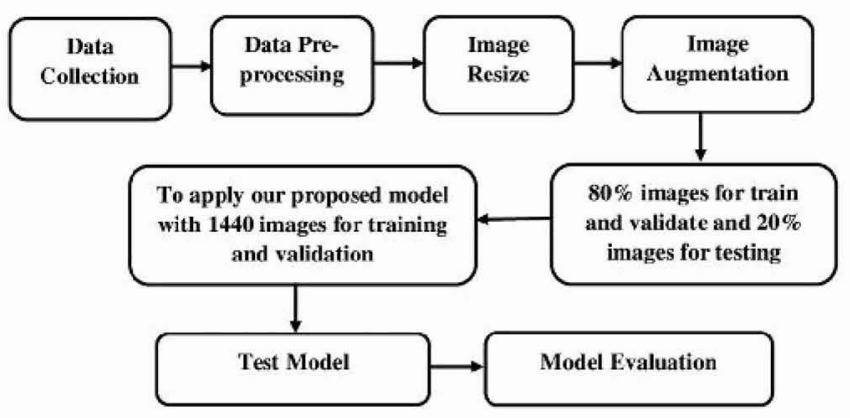

Fig. 1. The system architecture
In our experiment, we have used TensorFlow [20] and Keras [21] in the backend.

TensorFlow [20] is an open source software library which is useful for numerical calculations. It was created by Google scientists and specialists. It is useful for numerical calculations with mathematical expressions using information flow graphs. In such a graphs, edges represent the multidimensional data array when nodes represent mathematical operations.

Keras [21] is an application programming interface of the high-level neural network system written in Python. It is able to operate with TensorFlow [20], the CNTK, or the Theano. In order to work smoothly for image and text information, there are numerous applications for common usage of neural network building blocks like the layers, activation function, optimization, and many tools.

We have used a number of layers of Convolution Neural Network [16] like convolution layers, pooling layers, fully connected layers, activation function.

- Convolution is the principal layer that discovers highlights from an information picture. Convolution Input Data spares connections between pixels by learning the picture highlights utilizing tiny squares. This is a scientific task that accepts two contributions as Image Matrix and a channel or portion.

- On the off chance that the pictures are excessively expansive, pooling layers segmentation would lessen the number of parameters. Extraordinary pooling is called subsampling or downsampling, which diminishes the greatness of each guide, but preserves the vital data. There might be a few kinds of spatial pooling like Max Pooling, Average Pooling, and Sum Pooling. From the transformed component map, Max Pooling consistently takes the biggest component. Taking the biggest component can likewise take the normal pooling. Total of all the components in the element map constitutes the "Whole Pooling".

- In the Fully-Connected (FC) layer in our model, we complimented our matrix vector and feed it similar to a neural network into a fully connected layer.

- We use an activation function to make our output non-linear. In case of a Convolution Neural Network [16], the output of the functionality will be passed through the activation function. This may be the ReLU (Rectified Linear Unit) activation function. The output is shown in (1).

$$
f(x)=\max (0, x)
$$

\section{A. Dataset}

Our experiment usually requires large datasets of Acne images. Despite being a highly difficult task of getting these datasets with any ease, we have managed images for 5 classes of Acne diseases from dermnet.com. These are, Acne Closed Comedo, Acne Cystic, Acne Keloidalis, Acne Open Comedo, and Acne Pustular. The total number of images for our experiment stands at 1800 and per class it is 360 images. 


\section{B. Data Preprocessing}

For fewer data, the issue of 'Overfitting' plague the model. Overfittig simply means, the model attunes too closely to the training data, but may not perform well against unused test data. Again when we collect the data from the web, it has been observed that not all images are appropriately sized. To tackle these issues, first of all, we have resized the images to $224 \times 224$ pixel. Then we have applied 5 augmented methods on all the to 1800 images to get around overfitting. These methods are: rotate right +30 degrees, rotate left -30 degrees, flip horizontally, shading and translation. Fig. 2 visualizes a sample of the images. Here, $\boldsymbol{a c}, \boldsymbol{a c c}, \boldsymbol{a k}, \boldsymbol{a o c}$ and $\boldsymbol{a p}$ represent Acne Cystic, Acne Closed Comedo, Acne Keloidalis, Acne Open Comedo and Acne Pustular respectively.
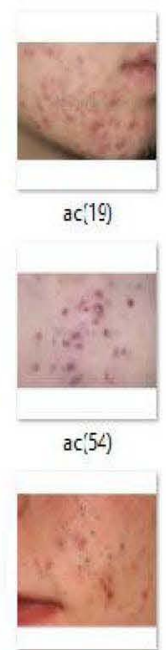

$\operatorname{acc}(52)$

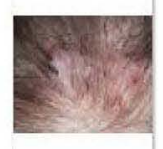

$\left.a k^{\prime} 39\right)$

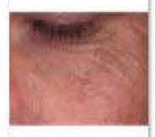

$\operatorname{aoc}(51)$

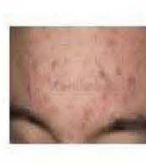

ac(22)

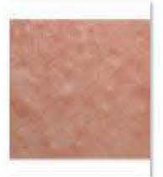

$\operatorname{acc}(2)$

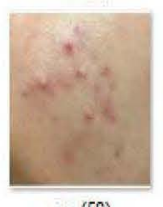

$\operatorname{acc}(58)$
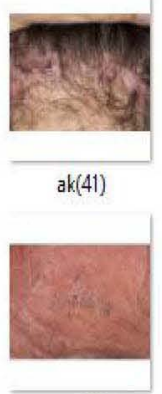

$\operatorname{aoc}(56)$

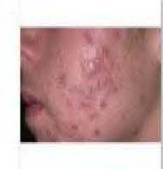

ax(29)

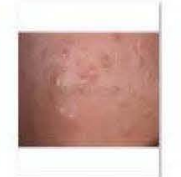

$\operatorname{acc}(12)$

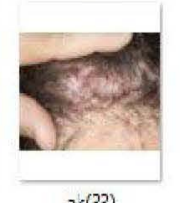

$\operatorname{ac}(33)$

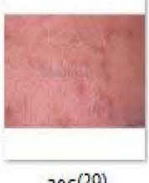

$\operatorname{aoc}(29)$

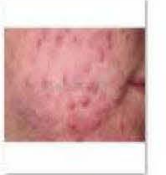

ao( $(2)$

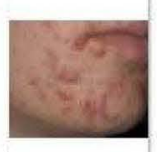

$\operatorname{ac}(31)$

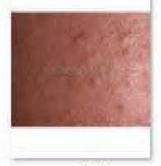

$\operatorname{arc}(21)$

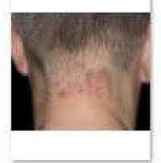

$i k(34)$
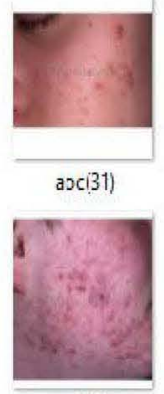

ap(54)
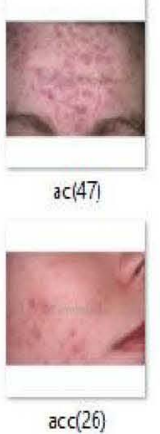

$\operatorname{acc}(26)$

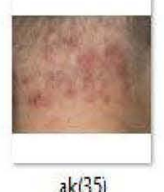

$\mathrm{ak}(35)$

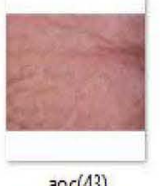

$\operatorname{aoc}(43)$

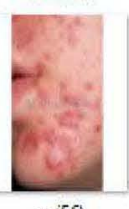

арі56)
Fig. 2. The sample of our dataset.

\section{Proposed Model}

After preprocessing we have used our proposed model "AcneNet", consisting of 47 convolution layers, some regularization methods like batch normalization and dropout, pooling layers, activation function, flatten layers and finally fully connected layer. The convolutional layer is the main building block of CNN [16]. To produce a feature map, the convolution layer works on the input data using filters or the kernel. The convolution shape for each layer is $64,128,256,512,1024$ and 2048 respectively. This feature map is also called 'Bottleneck Features" which is generated by the pooling layers. At the time of generating this, the last 1000 fully connected softmax layers have been ignored. This bottleneck features will be the ultimate prediction, which is used for a top classified training of our "AcneNet" images. This softmax layer with thousands of parameters allow us to reduce the time of training as well as to establish a very deep CNN for acne image detection problem.

We have used the RELU activation function with the same padding. The size of the pooling layer is $2 \times 2$. We have used 2 as the value of the stride. Stride denotes the pixel shift number on the string input matrix. When the stride is 2, we can move the filters in two pixels at once. The "Categorical_crossentropy" has been employed as a loss function because we have conducted multiple class. This function actually works at a time of training and have provided an average loss of our model, besides showing this loss in every step of the epoch as a validation loss. Then the batch normalization is done after each fully linked layer but after the occurrence of activation function and dropout.

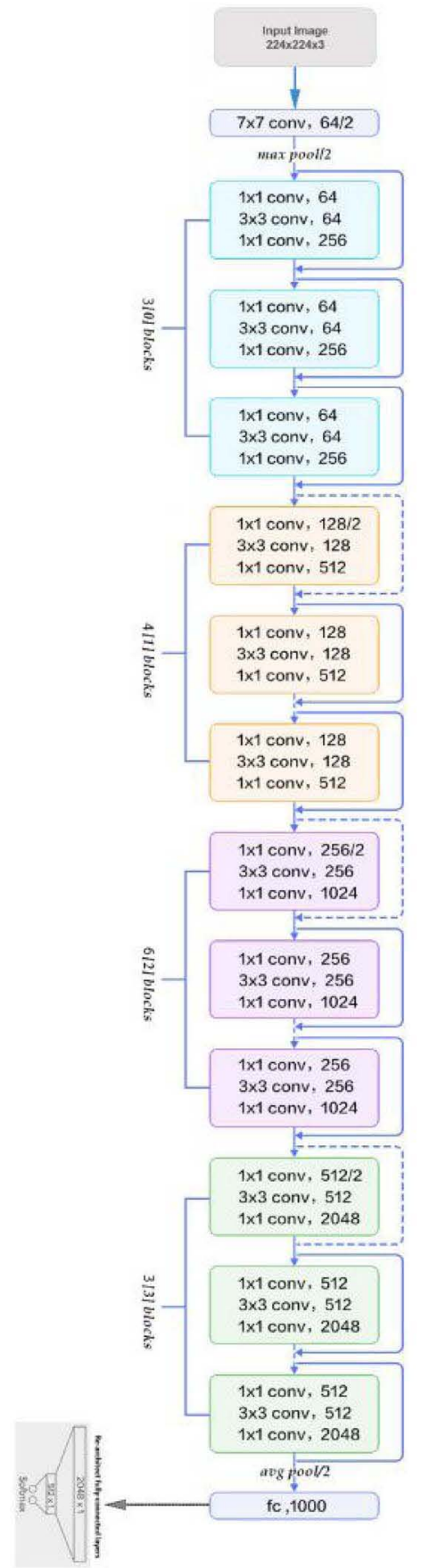

Fig. 3. The proposed model "AcneNet" architecture 
In our model, the dropout is 0.50 . All dropout layers are used for reducing overfitting. A single fully connected layer can receive only 1-dimensional data, which prompted us to 'Flatten' layers to convert 3D data into 1D. There are two dense functions, both encompasses the 'ReLU' activation function including size 2048 and 512. Following the application of dense function, we have used "softmax function" to be an output layer. Fig. 3 depicts the architecture of our proposed model.

\section{Training the Model}

Before training a neural network, it needs to be compiled with the correct cost function using the correct optimizer. In our proposed method, we used ADAM Optimizer [18]. ADAM optimization algorithm is computationally reasonable, facilitates implementation, effective and does not require high memory capacity. To fit the model we have used a batch size of 16 .

The proposed model has been applied to the acne dataset and obtained a good result on train, test, and validation sets. The training dataset is the sample of data used to make the model competent. The validation dataset is the data samples used to provide a model's neutral assessment during training dataset while tuning model hyperparameters. The test dataset is the data sample training used to provide a neutral assessment to fit a final model on the dataset. In our experiment, $80 \%$ of data we have used for training and validation and $20 \%$ data used for testing. That means we have a total of 1800 images. So 1440 images have used for training and validation and 360 images used for testing. Now each class contains 360 images.

\section{E. Confusion Matrix}

A Confusion Matrix [19] is a table that is often used to describe the performance of a classification model in test data for which the actual values are known. A confusion matrix [8] for a binary classifier reports the number of false positive (FP), false negative (FN), true positive (TP), and true negative (TN). In the case of multiple classes, there can be Confusion Matrix of $n \times n(n>2)$ levels. It contains $\mathrm{n}$ rows, $n$ columns and total $n \times n$ entries in the confusion matrix [8]. From these matrices, the number of FP, FN, TP, and TN cannot be counted directly. According to this method, classes for FP, FN, TP, and TN are calculated as par (2), (3), (4) and (5).

$$
\begin{gathered}
T P_{i}=a_{i i} \\
F P_{i}=\sum_{j=1, j \neq i}^{n} a_{j i} \\
F N_{i}=\sum_{j=1, j \neq i}^{n} a_{i j} \\
T N_{i}=\sum_{j=1, j \neq i}^{n} \sum_{k=1, k \neq i}^{n} a_{j k}
\end{gathered}
$$

We have made a Confusion Matrix (CM) [19] for our proposed model. We have also calculated Precision, Recall, Accuracy, F1-score, Sensitivity, and Specificity from CM
[19]. For calculating this we have used the (6) - (11) respectively.

$$
\begin{gathered}
\text { Accuracy }=(\mathrm{TP}+\mathrm{TN}) /(\mathrm{TP}+\mathrm{FP}+\mathrm{FN}+\mathrm{TN}) \\
\text { Precision }=\mathrm{TP} /(\mathrm{TP}+\mathrm{FP}) \\
\text { Recall }=\mathrm{TP} /(\mathrm{TP}+\mathrm{FN})
\end{gathered}
$$

F1 scores $=2 *($ Recall $*$ Precision $) /($ Recall + Precision $)$

$$
\begin{aligned}
& \text { Sensitivity }=\mathrm{TP} /(\mathrm{TP}+\mathrm{FN}) \\
& \text { Specificity }=\mathrm{TN} /(\mathrm{TN}+\mathrm{FP})
\end{aligned}
$$

\section{RESUlt ANALYSIS}

Table I is showing the Confusion Matrix [19] of our proposed model and we have already described above how it

\begin{tabular}{|c|c|c|c|c|c|c|}
\hline $\begin{array}{c}\text { Acne } \\
\text { Diseases }\end{array}$ & $\begin{array}{l}\text { Preci- } \\
\text { sion }\end{array}$ & Recall & $\begin{array}{l}\text { Accu- } \\
\text { racy }\end{array}$ & $\begin{array}{l}\text { F1- } \\
\text { Score }\end{array}$ & $\begin{array}{l}\text { Sensi- } \\
\text { tivity }\end{array}$ & $\begin{array}{l}\text { Spec- } \\
\text { ificity }\end{array}$ \\
\hline $\begin{array}{c}\text { Acne } \\
\text { Closed } \\
\text { Comedo }\end{array}$ & $79.17 \%$ & $96.61 \%$ & $95.28 \%$ & $87.02 \%$ & $96.61 \%$ & $95.02 \%$ \\
\hline $\begin{array}{l}\text { Acne } \\
\text { Cystic }\end{array}$ & $91.67 \%$ & $89.19 \%$ & $96.11 \%$ & $90.41 \%$ & $89.19 \%$ & $97.90 \%$ \\
\hline $\begin{array}{c}\text { Acne } \\
\text { Keloidalis }\end{array}$ & $98.61 \%$ & $98.61 \%$ & $99.44 \%$ & $98.61 \%$ & $98.61 \%$ & $99.65 \%$ \\
\hline $\begin{array}{c}\text { Acne } \\
\text { Open } \\
\text { Comedo }\end{array}$ & $97.22 \%$ & $79.55 \%$ & $94.44 \%$ & $87.50 \%$ & $79.55 \%$ & $99.26 \%$ \\
\hline $\begin{array}{l}\text { Acne } \\
\text { Pustular }\end{array}$ & $81.94 \%$ & $88.06 \%$ & $94.17 \%$ & $84.89 \%$ & $88.06 \%$ & $95.56 \%$ \\
\hline
\end{tabular}
can be calculated.

TABLE I. CONFUSION MATRIX OF OUR PROPOSED MODEL

\begin{tabular}{|c|c|c|c|c|c|}
\hline \hline $\begin{array}{c}\text { Acne } \\
\text { Diseases }\end{array}$ & $\begin{array}{c}\text { Acne } \\
\text { Closed } \\
\text { Comedo }\end{array}$ & $\begin{array}{c}\text { Acne } \\
\text { Cystic }\end{array}$ & $\begin{array}{c}\text { Acne } \\
\text { Keloidalis }\end{array}$ & $\begin{array}{c}\text { Acne } \\
\text { Open } \\
\text { Comedo }\end{array}$ & $\begin{array}{c}\text { Acne } \\
\text { Pustular }\end{array}$ \\
\hline $\begin{array}{c}\text { Acne } \\
\text { Closed } \\
\text { Comedo }\end{array}$ & 57 & 0 & 0 & 13 & 2 \\
\hline $\begin{array}{c}\text { Acne } \\
\text { Cystic }\end{array}$ & 0 & 66 & 0 & 1 & 5 \\
\hline $\begin{array}{c}\text { Acne } \\
\text { Keloidalis }\end{array}$ & 0 & 0 & 71 & 0 & 1 \\
\hline $\begin{array}{c}\text { Acne Open } \\
\text { Comedo }\end{array}$ & 2 & 0 & 0 & 70 & 0 \\
\hline $\begin{array}{c}\text { Acne } \\
\text { Pustular }\end{array}$ & 0 & 8 & 1 & 4 & 59 \\
\hline \hline
\end{tabular}

Table II displays the Precision, Recall, Accuracy, F1Score, Sensitivity, and Specificity of our model. We have calculated these from Confusion Matrix.

TABLE II. The Precision, ReCALl, ACCURACy, F1-SCORE, SENSITIVITY AND SPECIFICITY OF OUR MODEL 
After 20 epochs, the proposed model achieved the training accuracy of $86.28 \%$, the validation accuracy was scored at $86.11 \%$ and the testing accuracy at $95.89 \%$ on our Acne dataset. After analyzing the Confusion Matrix and the epoch table (Table III), we can say that our proposed model performed well on our dataset in training, validation and also in testing. Table III projects performance of 20 epochs (arranged by rows)

TABLE III. The Performance of EAch Epoch of OUR Proposed MODEL

\begin{tabular}{|c|c|c|c|c|}
\hline Epoch & Train Loss & $\begin{array}{c}\text { Train } \\
\text { Accuracy }\end{array}$ & $\begin{array}{c}\text { Validation } \\
\text { Loss }\end{array}$ & $\begin{array}{l}\text { Validation } \\
\text { Accuracy }\end{array}$ \\
\hline 1 & 1.5761 & 0.4115 & 0.8199 & 0.6944 \\
\hline 2 & 1.0194 & 0.5755 & 0.7235 & 0.7257 \\
\hline 3 & 0.8648 & 0.6493 & 0.7399 & 0.7014 \\
\hline 4 & 0.7588 & 0.6944 & 0.5248 & 0.8090 \\
\hline 5 & 0.7086 & 0.7109 & 0.5162 & 0.7743 \\
\hline 6 & 0.7039 & 0.7153 & 0.5263 & 0.7778 \\
\hline 7 & 0.6364 & 0.7465 & 0.4709 & 0.7986 \\
\hline 8 & 0.5792 & 0.7752 & 0.4545 & 0.8333 \\
\hline 9 & 0.5526 & 0.7969 & 0.4554 & 0.7986 \\
\hline 10 & 0.5100 & 0.8030 & 0.3603 & 0.8507 \\
\hline 11 & 0.4818 & 0.8108 & 0.3860 & 0.8507 \\
\hline 12 & 0.4828 & 0.8108 & 0.3418 & 0.8507 \\
\hline 13 & 0.4623 & 0.8142 & 0.3506 & 0.8542 \\
\hline 14 & 0.4517 & 0.8290 & 0.3553 & 0.8472 \\
\hline 15 & 0.4266 & 0.8325 & 0.3331 & 0.8542 \\
\hline 16 & 0.3947 & 0.8646 & 0.3061 & 0.8924 \\
\hline 17 & 0.3815 & 0.8602 & 0.3735 & 0.8438 \\
\hline 18 & 0.3763 & 0.8628 & 0.3071 & 0.8681 \\
\hline 19 & 0.3623 & 0.8568 & 0.4432 & 0.8229 \\
\hline 20 & 0.3521 & 0.8628 & 0.2964 & 0.8611 \\
\hline
\end{tabular}

Fig. 4 represents the training accuracy and the validation accuracy graph of our model. Here the green line represents the performance of the validation accuracy and the blue line represents the training accuracy. Besides, X-axis represents each epoch while Y-axis represents accuracy. 20 epochs signify they have gone over all of the training data 20 times. In deep learning models, an epoch is a hyperparameter. It is defined before the beginning of training a model. One epoch literally means a complete dataset has been passed both forward and backward through the neural network only once.

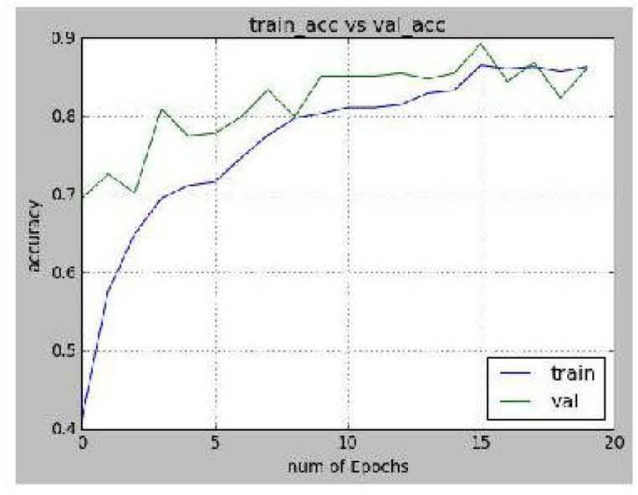

Fig. 4. The graph of the training accuracy vs the validation accuracy.

Fig. 5 represents the training loss and the validation loss graph of our model. Here the green line represents the performance of the validation loss and the blue line represents the training loss; while $\mathrm{X}$-axis represents each epoch while $\mathrm{Y}$-axis represents the loss.

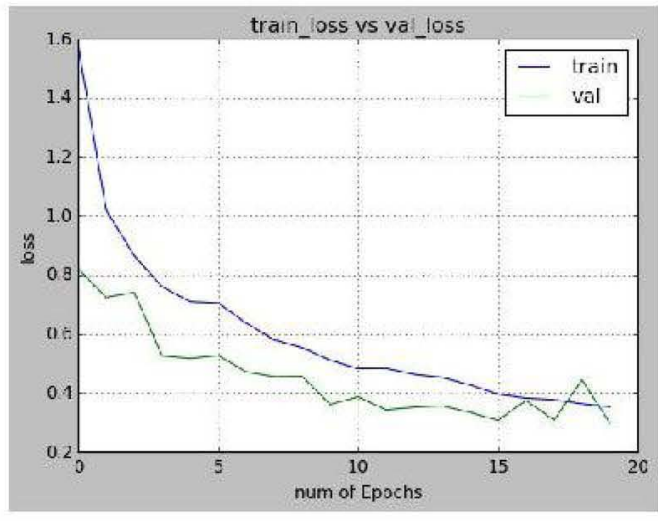

Fig. 5. The graph of the training loss vs the validation loss.

We have also applied Inception-V3 [14] and MobileNet [15] version of CNN [16] on our "AcneNet" dataset. These models have likewise performed incredibly well except creating the overfitting, (although shown great accuracy). The accuracy and cross-entropy graph of Inception-V3 [14] and MobileNet [15] were given below respectively. For both of the versions of CNN we have used 5000 training steps.
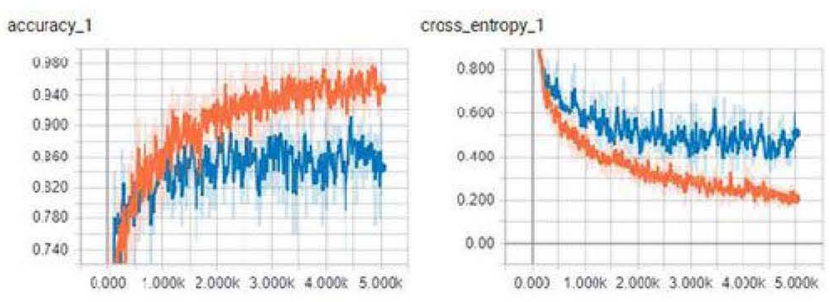

Fig. 6. The graph of the accuracy and the cross-entropy graph of InceptionV3.
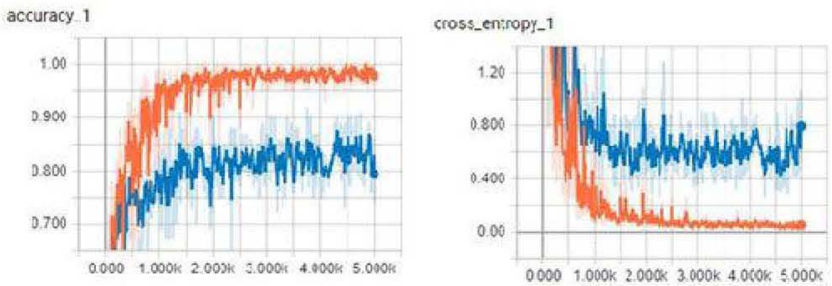

Fig. 7. The graph of the accuracy and the cross-entropy graph of MobileNet. 
Table IV outlines a comparison with other CNN [16] models like Inception-V3 [14] and MobileNet [15]. From Table IV, we can say that the proposed model, "AcneNet", has performed significantly better in comparison to other models. Both Inception-V3 and MobileNet are quite popular. In order to get a precious result, we have used more layers with legion parameters than other models of CNN. As this is a subtle work so we had to go much deeper to classify the images through layers. Otherwise, the classes create a conflict between them. Therefore, we had to spend a bit more time than others as well.

TABLE IV. COMPARISON BETWEEN OUR PROPOSED MODEL AND OTHER CNN MODELS

\begin{tabular}{|c|c|c|c|c|c|}
\hline \hline $\begin{array}{c}\text { Model } \\
\text { Name }\end{array}$ & $\begin{array}{c}\text { Top-1 } \\
\text { Accuracy }\end{array}$ & $\begin{array}{c}\text { Top-5 } \\
\text { Accuracy }\end{array}$ & $\begin{array}{c}\text { Training } \\
\text { Accuracy }\end{array}$ & $\begin{array}{c}\text { Testing } \\
\text { Accuracy }\end{array}$ & $\begin{array}{c}\text { Param- } \\
\text { eters }\end{array}$ \\
\hline $\begin{array}{c}\text { Inception- } \\
\text { V3 }\end{array}$ & $78.80 \%$ & $94.40 \%$ & $85.5 \%$ & $91.33 \%$ & $\begin{array}{c}23,851, \\
784\end{array}$ \\
\hline MobileNet & $66.51 \%$ & $87.12 \%$ & $83.2 \%$ & $92.45 \%$ & $\begin{array}{c}4,253,8 \\
64\end{array}$ \\
\hline $\begin{array}{c}\text { AcneNet } \\
\text { (Proposed } \\
\text { model) }\end{array}$ & $\mathbf{6 8 . 0 9 \%}$ & $\mathbf{8 8 . 3 1 \%}$ & $\mathbf{8 6 . 1 1 \%}$ & $\mathbf{9 5 . 8 9 \%}$ & $\begin{array}{c}\mathbf{2 5 , 6 3 6} \\
\mathbf{7 1 2}\end{array}$ \\
\hline \hline
\end{tabular}

From Table IV, it can be observed that we have achieved higher accuracy from other models of CNN because we have used an extra layer. But in CNN [16], there is no opportunity to use more and more layer to improve the accuracy because CNN is a plain network. But in our model, we have used the identified block (shortcut connections) so that we can go significantly deeper to classify the images really precisely. In $\mathrm{CNN}$, we have to go straight if we have similar layers. No layers can be avoided. But in our model, we can jump from one layer to another using identify block which means similar layers can be skipped.

\section{CONCLUSION AND FUTURE WORK}

The research work presented in this paper conceived a new CNN [16] model that performs better classification accuracy (95.89\%) than other related models. The model also needed lesser training data, training and validation time in comparison to other related CNN based models built for acne identification.

Even though our system has performed admirably, but in future we would like to collect more resources to design a more complete architecture with increased number of layers so that we can improve upon the current level of performance.

\section{REFERENCES}

[1] https://www.health24.com/Medical/Acne/About-acne/Acne20120721, Accessed: 30 May, 2019.

[2] Suva, M., "A Brief Review on Acne Vulgaris: Pathogenesis, Diagnosis, and Treatment", Research \& Reviews: Journal of Pharmacology. Vol. 4, Issue 3, 2015.

[3] https://www.medicalnewstoday.com/articles/107146.php, Accessed: 31 May, 2019.

[4] Taylor, M., Gonzalez, M. and Porter, R., "Pathways to Inflammation: Acne Pathophysiology”, Eur J Dermatol. 21(3), 323-33p, 2011.

[5] Vos, T. F. Years Lived with Disability (YLDs) for 1160 Sequelae of 289 Diseases and Injuries 1990-2010: A Systematic Analysis for the Global Burden of Disease Study 2010, Lancet, 380(9859): 2163-96p, 2012.
[6] Razavian, N., Sontag, D., "Temporal Convolutional Neural Networks for Diagnosis from Lab Tests", Computer Science Department, New York University, 2015.

[7] Maroni, G., Ermidoro, M. and Previdi, F., "Automated Detection, Extraction and Counting of Acne Lesions for Automatic Evaluation and Tracking of Acne Severity", Department of Management, Information and Production Engineering, 2017.

[8] Shen, X., Zhang, J., Yan, C., \& Zhou, H. An Automatic Diagnosis Method of Facial Acne Vulgaris Based on Convolutional Neural Network. Scientific reports, 8(1), 2018.

[9] Chantharaphaichit, T., Uyyanonvara, B., Sinthanayothin, C., and Nishihara, A. Automatic acne detection with featured Bayesian classifier for medical treatment. Proceedings of the 3rd International Conference on Robotics, Informatics, and Intelligence Control Technology (RIIT2015), 10-16, 2015.

[10] Digitale Photographie GmbH, "VISIA Complexion Analysis", http://www.visia-complexion-analysis.com/visia-complex ionanalysis.asp, 2004.

[11] Fujii, H., Yanagisawa, T., Mitsui, M., et al., "Extraction of acne lesion in acne patients from Multispectral Images," 30th Annual International IEEE EMBS Conference, pp. 4078-4081, 2008.

[12] Alamdari, N., Alhashim, M., and Fazel-Rezai, R. "Detection and classification of acne lesions in acne patients: a mobile application", 2016 IEEE International Conference on Electro Information Technology (EIT), 739-743, 2016

[13] Phillips S.B., Kollias N, Gillies R, Muccini J.A. and Drake L.A., "Polarized light photography enhances visualization of inflammatory lesions of acne vulgaris," Journal of the American Academy of Dermatology, pp. 948-952, 1997.

[14] Szegedy, C., Liu, W., Jia, Y., Sermanet, P., Reed, S., Anguelov, D., Erhan, D., Vanhoucke, V. and Rabinovich, A., "Going deeper with convolutions", arXiv:1409.4842v1, 2014

[15] Andrew G., Menglong, H., Bo, Z., Kalenichenko, C.D., Wang, W., Weyand, T., Andreetto, M. and Adam, H., "MobileNets: Efficient Convolutional Neural Networks for Mobile Vision Applications", arXiv: 1704.04861v1, 2017.

[16] Krizhevsky, A., Sutskever, I., Hinton, G.E., "ImageNet Classification with Deep Convolutional Neural Networks", Communications of the ACM CACM Homepage archive Volume 60 Issue 6, pp. 84-90, 2017.

[17] He, K., Zhang, X., Ren, S. and Sun, J., "Deep Residual Learning for Image Recognition”, arXiv: 1512.03385v1, 2015.

[18] Diederik P. Kingma, Jimmy Lei Ba, "ADAM: A METHOD FOR STOCHASTIC OPTIMIZATION”, arXiv: 1412.6980v9 [cs.LG] 30 Jan 2017.

[19] David, N., Lior, M. and Shmilovici, R.A., "Using the Confusion Matrix for Improving Ensemble Classifiers", IEEE 26-the Convention of Electrical and Electronics Engineers, Israel, 2010.

[20] Martín Abadi, Paul Barham, Jianmin Chen, Zhifeng Chen, Andy Davis, Jeffrey Dean, Matthieu Devin, Sanjay Ghemawat, Geoffrey Irving, Michael Isard, Manjunath Kudlur, Josh Levenberg, Rajat Monga, Sherry Moore, Derek G. Murray, Benoit Steiner, Paul Tucker, Vijay Vasudevan, Pete Warden, Martin Wicke, Yuan Yu, and Xiaoqiang Zheng, Google Brain, "TensorFlow: A System for Large-Scale Machine Learning", 12th USENIX Symposium on Operating Systems Design and Implementation (OSDI '16). November 2-4, 2016.

[21] Vidnerova, P., Neruda, R., "Evolving Keras architectures for sensor data analysis", Federated Conference on Computer Science and Information Systems (FedCSIS), 2017.

[22] Hoon, K. S., Yeo, K. C., Azam, S., Shunmugam, B. and Boer, F. D., "Critical review of machine learning approaches to apply big data analytics in DDoS forensics" 2018 International Conference on Computer Communication and Informatics (ICCCI), 2018.

[23] Vokerla, R. R., Shanmugam, B., Azam, S., Karim, A., Boer, F. D., Jonkman, M. and Faisal, F., "An Overview of Blockchain Applications and Attacks" International Conference on Vision Towards Emerging Trends in Communication and Networking (ViTECoN), 2019.

[24] Khan, M. I., Faisal, F., Azam, S., Karim, A., Shanmugam, B. and Boer, F. D., "Using Blockchain Technology for File Synchronization", IOP Conference Series: Materials Science and Engineering, 2019, (In Press). 